\title{
Technologic optimization of a virtual disease focused panel during the COVID pandemic and beyond
}

\author{
Mohammed Saleh $^{1}$ Priya Bhosale ${ }^{1} \cdot$ Dheeraj Reddy Gopireddy $^{2} \cdot$ Malak Itani $^{3} \cdot$ Samuel Galgano ${ }^{4}$. \\ Ajaykumar Morani ${ }^{1,5}$
}

Received: 2 January 2021 / Revised: 19 February 2021 / Accepted: 25 February 2021 / Published online: 16 March 2021

(c) The Author(s), under exclusive licence to Springer Science+Business Media, LLC, part of Springer Nature 2021

\begin{abstract}
Since the beginning of the COVID-19 pandemic, several service fields have opted for virtual work as much as possible, in order to decrease the spread of the virus. Although several articles have now addressed the key issues in telecommunications and medical education, none have described the digital or virtual functionality of a professional society disease focused panel (DFP) or inter-institutional collaborations. This is extremely important as we believe that the virtual functioning of the DFP will be the new norm for years to come. In this article, we highlight the limitations in the functioning of DFP brought forth due to the ongoing pandemic, while also providing the digital technologic solutions to adapt and also maintain or increase its productivity.
\end{abstract}

Keywords Pandemic $\cdot$ COVID-19 $\cdot$ DFP $\cdot$ Virtual $\cdot$ Digital $\cdot$ SAR

\section{Introduction}

The Society of Abdominal Radiology (SAR) mentors panels that include expert members from different disciplines and fields to conjointly form guidelines to optimize the diagnosis and management of specific diseases through clinical, educational, and research collaborations. Specifically, the SAR DFPs optimize medical education and research by bringing together radiology and non-radiology physician experts in a specific disease field. Success of a disease focused panel (DFP) relies heavily on the interaction among its members and the formation of solid interpersonal relationships through meeting platforms provided by annual physical meetings and respective institutional multidisciplinary conferences. These however, have been heavily restricted and compromised by the ongoing unprecedented Coronavirus disease 2019 (COVID-19) pandemic. Just like the effect on medical services and education, the functionality of DFPs have also been compromised by the pandemic. The pandemic has led to several challenges in the development and collaboration of DFPs due to restricted physical meetings, limited travel and almost altogether cancellations of physical radiology society conferences in lieu of virtual meetings. This in turn could hinder medical advancements and discoveries dependent on collaborations. However, several technological advances and innovations have provided us

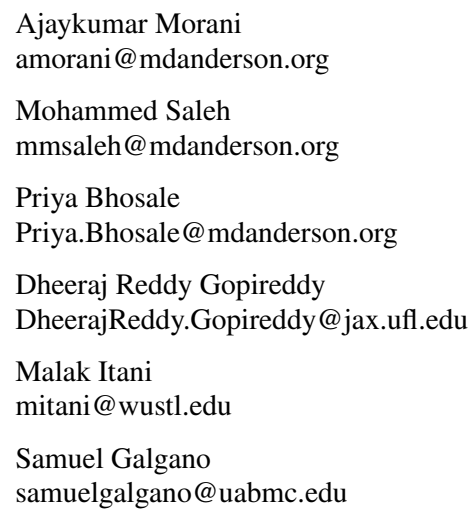

1 Department of Diagnostic Radiology, The University of Texas MD Anderson Cancer Center, Houston, TX 77030 USA

2 Department of Diagnostic Radiology, The University of Arizona Health Sciences, Tucson, AZ 85721, USA

3 Mallinckrodt Institute of Radiology, Washington University in St. Louis, St. Louis, MO 63110, USA

4 Department of Radiology, University of Alabama at Birmingham, Birmingham, AL 35243, USA

5 Department of Abdominal Radiology, The University of Texas MD Anderson Cancer Center, Houston, TX 77030, USA 
with opportunities to counter the limitations set forth by the virus [1], and adapt to maintain the functionality of the DFP by implementing measures for an uninterrupted educational experience $[2,3]$. This article aims at describing the problems faced in initiating and operationalizing our DFP during COVID-19 pandemic, and also describes some of the solutions which we applied to continue the growth of the panel.

\section{Pre-COVID operations}

\section{Annual meetings}

Prior to the COVID pandemic, DFP members primarily met during the major annual meetings such as Radiological Society of North America (RSNA) and SAR. Large conference rooms were reserved at the meeting venues for physical face to face meetings with discussions of annual goals, major advances, and planning. The initial inaugural physical meeting was usually vital to introduce DFP members and perform icebreaking activities to familiarize members with one another, facilitate collaborations and discuss expectations and goals of the panel. Subsequent meetings were usually conducted via teleconferences and emails, which served as the major communication means for exchanging ideas and materials among members. Flow and sharing of data were also mainly through emails, or with limited use of cloud data storage systems.

Education through the DFP to SAR and non-SAR members has largely been in the form of lectures and CME programs physically delivered at various society meetings or invited physical lecturers at different institutions. Various group text or chat applications like WhatsApp (Facebook, Inc., Menlo Park, California) and Telegram (Telegram FZ LLC, London, United Kingdom) were often used and are still being used for group communication. To learn and spread the knowledge at a global scale, social media platforms, such as Twitter (Twitter, San Francisco, California), continue to be important tools [4] for communication.

\section{Post-COVID operations with technologic optimization}

\section{Background}

Established in the early weeks of pandemic at 2020 annual meeting, Neuroendocrine DFP is one of the newest SAR DFPs. It has 24 members and 14 consultants spanning 13 institutions from 12 different states in North America. We quickly adapted to limit the impact of the loss of physical meetings and face to face collaborations during the development of our DFP. The effects of the pandemic could be seen during SAR 2020 Annual Meeting. Unfortunately, some of the DFP members and chairs were not able to attend, either due to the risk of infection or due to institutional travel restrictions in addition to some expected absentees from other conflicts. A follow-up virtual conference was scheduled soon afterwards to discuss the deliverables, and much of the DFP's further growth happened via emails and virtual meeting collaborations due to quick digital adaptation of the panel.

\section{Virtual meetings}

During the current COVID-19 pandemic, the need for novel tools to optimize virtual collaborations have accelerated. Many institutions are trying to improvise on safe functioning and learning while practicing social distancing with use of new technologies such as Webex (Cisco systems, Milpitas, California), Zoom (Zoom Video Communications, San Jose, California), Microsoft Teams (Microsoft, Redmond, Washington), and Google Meet (Google, Mountain View, California) [4-10]. These tools are easily accessible due to their cross platform functionality on several devices including desktops, laptops, tablets, and smart phones [5]. Several authors $[2,11]$ have described how web-based conferencing can be used to continue medical care and education while respecting social distancing. The DFP has adopted similar practices since meetings have become virtual (and may be more frequent) through the use of web-based platforms of Zoom and Cisco WebEx (Fig. 1). Screen sharing (Fig. 2) allows the presenter to share material with rest of the members $[12,13]$. Microsoft Teams' platform allows multiple functions including its ability to act as a cloud system with OneDrive (Microsoft, Redmond, Washington) similar to Google Drive (Google, Mountain View, California) where documents can be shared and simultaneously edited by several DFP members $[4,14,15]$.

These user-friendly platforms provide some social and virtual presence and means of digital collaboration [16]. Turning on video cameras by the members allows realtime face to face interactions and may provide more depth to the interpersonal connection which to some extent, may be similar to that of physical meetings. Virtual meetings also decrease the time spent physically arriving at a location, and the participants can access the meeting at their convenience. We implemented several features to maximize efficiency and interaction among the members during the meetings. Personal or group chatting, live polling to support audience response system (Fig. 3), as well as "raising hand" options have improved the smoothness of virtual meetings [4]. In fact, by implementing these tools, virtual meetings have become more fruitful than physical meetings by minimizing time lost due to travel or other commitments that may conflict with members' physical 
Fig. 1 Virtual framework of a Disease Focused Panel during the COVID pandemic. This figure shows some different common platforms that can be used to have successful virtual meetings
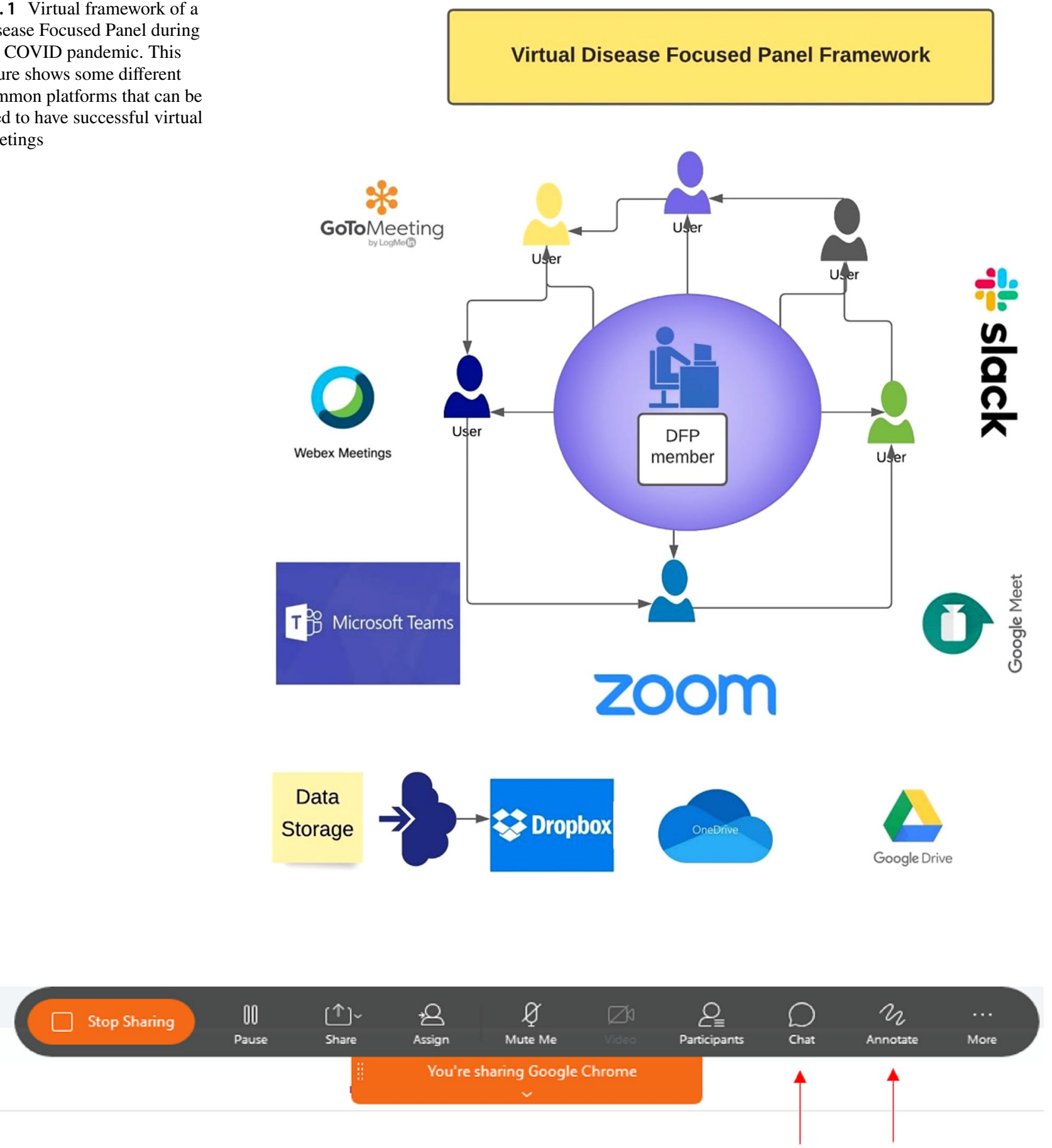

Fig. 2 An example of WebEx's screen sharing option. Interaction with the members is easy and effective both visually and audibly through messaging, annotation, and audio

presence while improving attendance at the same time due to accessibility. We believe that this may be the new norm for years to come given the relative convenience and increasing comfort with virtual format.

\section{Meeting minutes (recording and storing)}

Easy to use recording features on virtual platforms or recording of the virtual meetings using third party applications 
Fig. 3 An example of the polling option provided by Webex showing the possible answers to a specific question along with the results in percent and bar graph format as well as the time remaining for the poll

\section{Polling}

$[\downarrow]$

Poll in progress:

\section{Questions}

Results

Bar Graph

1. How well does virtual conferencing ...
A. Not well
B. Quite well
C. Very well

No Answer

$0 / 0(0 \%)$

$0 / 0(0 \%)$

$0 / 0(0 \%)$

$0 / 0(0 \%)$

\section{Polling status

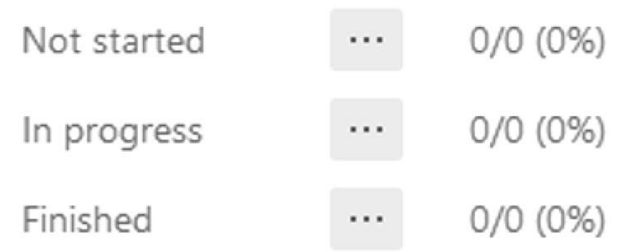

Remaining time: 4:46 Time limit: 5:00

New Poll
Edit Questions
Close Poll such as Snagit (TechSmith, Okemos, Michigan) and Zoom allow easy capturing of meeting minutes. These recordings can be stored on shared cloud storage system drives such as Box (Box, Redwood City, California), Dropbox (Dropbox Inc, San Francisco, California), OneDrive or Google Drive with access to all DFP members at their convenience [17]. This allows the panel member absentees to stay tuned to the DFP's latest advancements.

\section{Surveys}

Prior to the COVID pandemic, DFP members used to give in-person feedback following any meeting to improve future functionality. Getting feedback may be sometimes challenging during virtual meetings due to concomitant feedback or occasionally noise from forgetting to mute a call. In that case, the panel members can use the private or public chat box feature of the platform and share their views, questions and suggestions. This helps capture realtime feedback and necessary improvements can be implemented quickly and efficiently. Automatic post-meeting surveys can be built when scheduling the meetings or developed separately with independent vendors such as Qualtrics (Qualtrics XM, Seattle, WA and Provo, Utah). We have found that these surveys make it easier to rate how well the DFP is meeting its objectives, and also provide direct input on how to improve the DFP functionality and streamline virtual interactions. 
From the specific disease point of view, as different institutions may use different imaging protocols and practices, relevant questionnaires can be built and disseminated among the DFP members using shared spreadsheets or survey tools such as Google Forms (Google, Mountain View, California). We are in the midst of building such a questionnaire for information on imaging practices for neuroendocrine tumors. We anticipate that it will help us identify trends in imaging and clinical practice in various institutions throughout the country, understand the expertise each member brings and prepare guidelines to adapt best practices in the field. This provides a unique way of creating surveys by multiple members editing and revising questionnaire documents simultaneously with tracked changes or at individual's convenience without sacrificing security, fidelity and quality of data [18]. There is also no need to wait for feedback from one member before sending the document to the next member and so on, thus saving valuable time. This also avoids confusion of multiple revised versions of the documents through traditional email approach.

\section{Education}

One of the DFP's ultimate goals is to expand knowledge. We started this via case of the month and web-based lectures and webinars every 2nd month. A shared Google Spreadsheet was used for members to sign-up and contribute the cases to upload periodically on the SAR DFP webpage, which is accessible to the public. The lectures are given by expert clinicians, surgeons and radiologists to discuss imaging, staging/grading and multidisciplinary management of neuroendocrine tumors which help expand the members' knowledge. The virtual nature of these presentations make them more interactive as the small group can interject, ask questions or place questions in the chat box during the presentation. Thus, web lectures, webinars and even symposia can be conducted with contribution from the experts remotely, and they can be easily recorded, stored for future use, and/or disseminated for educational purposes. Even recorded video clips from multiple remote speakers can be edited, trimmed and merged together, to create joint lectures and presentations [19]. We created a similar joint symposium for SAR by optimizing and merging video clips of multiple multidisciplinary case presentations and lecture video clips of our 11 remote DFP speakers. Additionally, social media handles (like Twitter) can be used to disseminate the information, education and for updates on the latest advancements and discoveries in the field among DFP members, society and the community at large [4].

\section{Data sharing and research}

In order to maximize productivity during or after the pandemic, several shared digital technologic tools can be used to encourage collaborations among the DFP members for remote research [20]. In our case, we prepared a shared Google document for members to share research ideas remotely and share scientific articles they have published in the field of DFP interest. Multiple members could again access and edit the document at the same time, avoiding the delay of passing the document among members and avoiding multiple revised document versions among the 24 members and 14 consultants of our DFP. Shared documents on different platforms (e.g. Google Docs) can also be used to formulate a plan for writing manuscripts and white papers, where each member can sign up to write a section of their choice/ interest and expertise. These platforms can also be uniquely used to share and jointly write the manuscripts by remote multi-institutional authors simultaneously with tracked changes minimizing the redundancy in the paper. Users can also cite the references while editing the shared documents. For example, some citation management software like Paperpile (Paperpile LLC, Cambridge, MA), RefWorks (Proquest LLC, Ann Arbor, MI), Sciwheel (Sciwheel Limited, London, UK), Zotero (Zotero, Fairfax, VA), ReadCube Papers (ReadCube Papers Enterprise, Cambridge, MA) all support citations and bibliography management [21, 22] in Google Docs. Sometimes, this may need a work-around when using digital products from different vendors based on compatibility issues. For example, while using Endnote (Clarivate Analytics, Philadelphia) citation manager, travelling library can be shared and synced among multiple users, and references may need to be copied-pasted (or draggedpasted) to shared Google Doc for latter formatting of the citations in the Microsoft word document.

Virtual platforms such as zoom or WebEx can be easily used to share images for interpretation, education and research. The images must be deidentified on the PACS (Picture Archiving and Communication System) and presented, and the imaging findings can be interpreted and documented remotely. Interestingly, this can improve efficiency since different readers can work in parallel while being blinded to patient information and the other readers' results. Each individual reader can then decide to fill in scoring information either in common passcode and PHI protected database such as Research Electronic Data Capture (REDCap, Vanderbuilt University) or into an Excel spread sheet. Currently, our DFP is trying to establish a cloud storage system for creating a common shared imaging repository of HIPAA compliant de-identified image data sets for educational and research purposes, which may allow the collaborators to work independently on the imaging datasets at their convenience [23]. For DFP members naïve to this technology, research intern, 
fellow or designated member of the DFP can help co-ordinate with cloud system education and research.

\section{Limitations}

One of the main limitations in virtual meetings and operations is digital literacy. Members who are less tech savvy might find it difficult to transition completely virtually. Unfortunately, during this time of need, virtual communication was not a choice and all of radiology had to use telecommunication. For example, in a recent internal survey in our DFP, we found that almost all $(96 \%, n=25 / 26)$ of the responding participants learnt about some, several or all of the enquired digital conferencing platforms during the pandemic (Fig. 4), and 61\% ( $n=16 / 26)$ had some difficulty using these platforms in the early phase of the pandemic. About 46\% ( $n=12 / 26)$ had to sometimes even stop or postpone their presentation due to difficulty in navigating the platform (Fig. 5). Several methods have been implemented by vendors to simplify the use of their programs and make them user friendly, or through instructions ranging from written tutorials to walkthrough videos. About $23 \%$ of the respondents in our DFP survey needed to watch some kind of tutorial for use of one or the other digital platform. As reported by other studies, although some programs may require time to get adjusted, we observed that the learning curve was quick for the DFP as witnessed by the lack of difficulties in later meetings [24, 25]. Regarding multiinstitutional research among different authors, there may still be issues with all readers getting together at the same time for consensus studies, and may need lot of co-ordination. There may also be potential issues of HIPAA compliance and Institutional Review Board approvals, as with most other multi-institutional trials [26, 27]. Although these virtual platforms provide a social component, the differences in people's location can be distracting due to background noise or motion in a person's webcam [24]. When bothersome, this
Fig. 4 a, b Bar diagrams created from the results of internal survey among the members $(n=26)$ of a disease focused panel showing the prevalence of knowledge of various digital conferencing platforms in the members prior to a CPVID-19 (corona virus-19) pandemic and prevalence of new knowledge acquired about the platforms during $\mathbf{b}$ the ongoing CPVID19 pandemic
A

\section{Knowlege of digital platforms prior to COVID-19 pandemic}

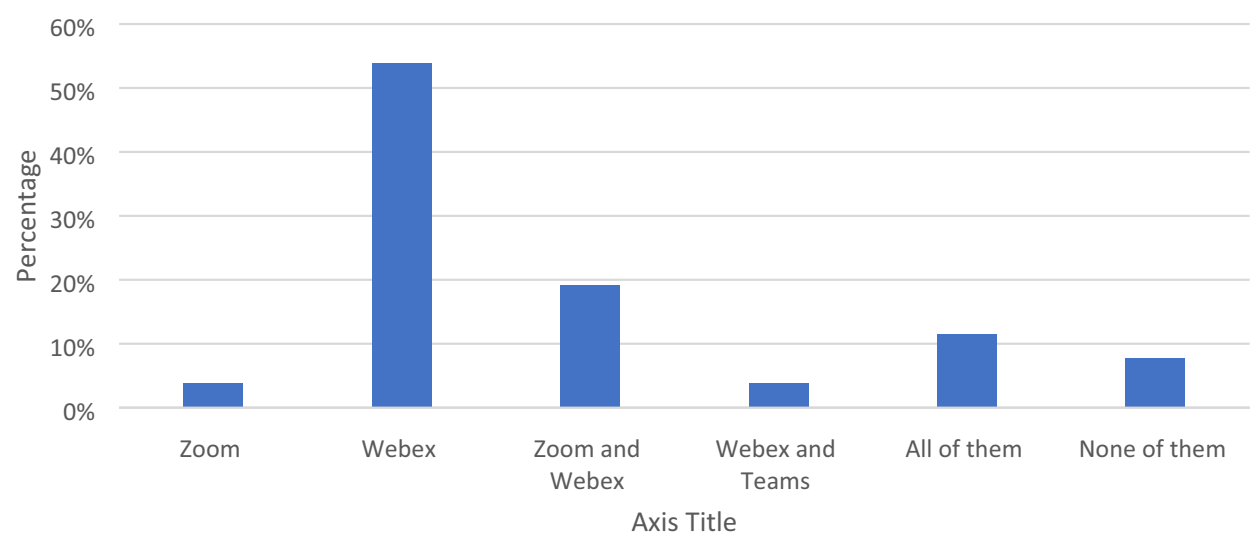

B

\section{New digital platform knowldge during COVID-19 pandemic}

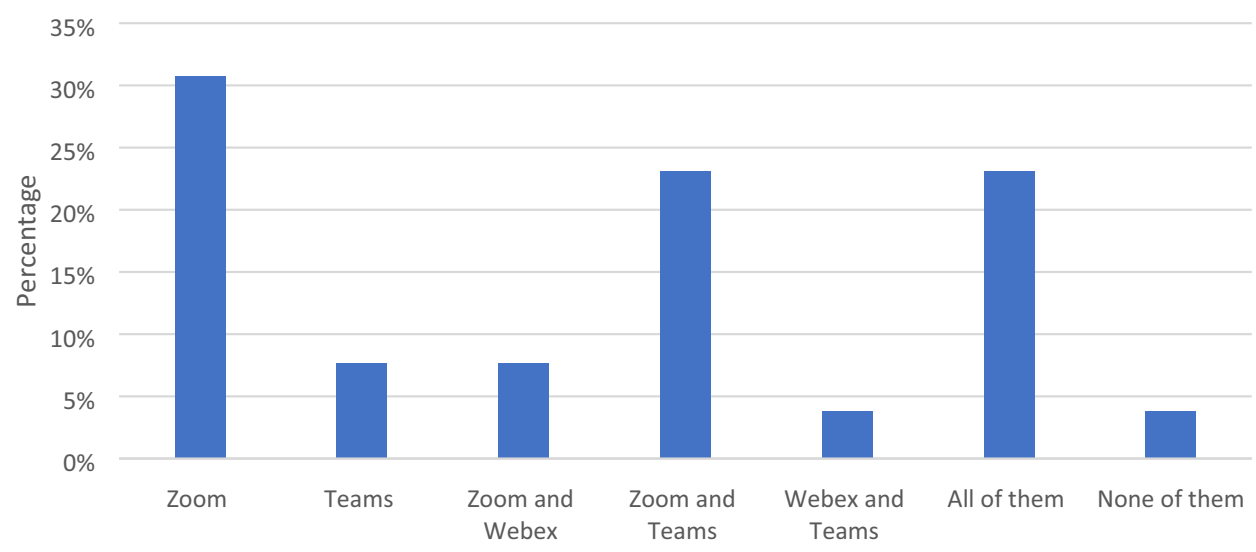


Fig. 5 Pie chart created from the results of internal survey in the disease focused panel showing frequency of stopping or postponement of their online meetings by the DFP members due to difficulty navigating the digital platforms in the early phase of corona virus pandemic
Stopped or postponed meeting due to difficulty nagivating the digital platforms

(Teams, Zoom \& WebEx )

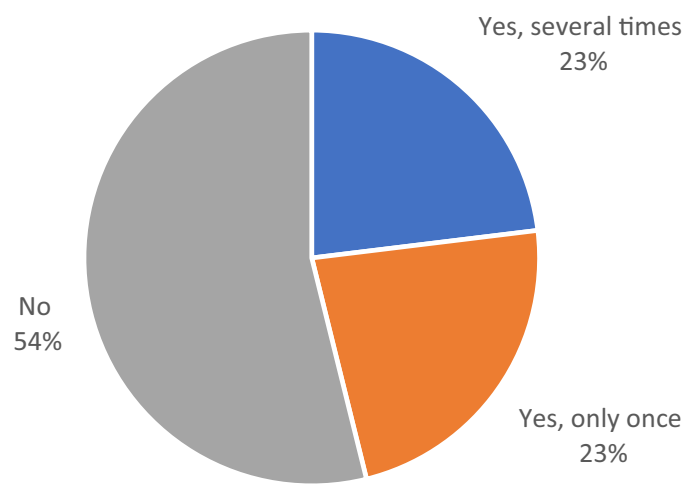

Acknowledgements SAR Neuroendocrine Disease Focused Panel.

Author contributions All authors equally contributed to this paper with conception and design of the study, literature review and analysis, drafting and critical revision and editing, and final approval of the final version.

Funding No financial support.

\section{Declarations}

Conflict of interest No potential conflicts of interest.

\section{References}

1. Tokuç B, Varol G. Medical education in Turkey in time of COVID-19. Balkan medical journal. 2020;37(4):180.

2. Virarkar M, Jensen C, Javadi S, Saleh M, Bhosale PR. Radiology Education Amid COVID-19 Pandemic and Possible Solutions. Journal of Computer Assisted Tomography. 2020;44(4):472.

3. Chick RC, Clifton GT, Peace KM, Propper BW, Hale DF, Alseidi AA, et al. Using technology to maintain the education of residents during the COVID-19 pandemic. Journal of Surgical Education. 2020.

4. Almarzooq Z, Lopes M, Kochar A. Virtual learning during the COVID-19 pandemic: a disruptive technology in graduate medical education. Journal of the American College of Cardiology; 2020.

5. Bhargava P, Lackey AE, Dhand S, Moshiri M, Jambhekar K, Pandey T. Radiology education 2.0-on the cusp of change: part 1. Tablet computers, online curriculums, remote meeting tools and audience response systems. Academic Radiology. 2013;20(3):364-72.

6. McRoy C, Patel L, Gaddam DS, Rothenberg S, Herring A, Hamm J, et al. Radiology Education in the Time of COVID-19: A Novel Distance Learning Workstation Experience for Residents. Academic radiology. 2020;27(10):1467-74.

7. Jena PK. Impact of Covid-19 on higher education in India. International Journal of Advanced Education and Research (IJAER). 2020;5. 
8. Mishra K, Boland MV, Woreta FA. Incorporating a virtual curriculum into ophthalmology education in the coronavirus disease-2019 era. Current opinion in ophthalmology. 2020;31(5):380-5.

9. Porpiglia F, Amparore D, Autorino R, Checcucci E, Cooperberg MR, Ficarra V, et al. Traditional and Virtual Congress Meetings During the COVID-19 Pandemic and the Post-COVID-19 Era: Is it Time to Change the Paradigm? European Urology. 2020.

10. Torres MG, Buck C, Gouldin C. Making the Leap from the Traditional to the Virtual Educational Experience. New England Journal of Higher Education. 2020.

11. Catanzano TM, Nandwana SB, Folio LR. Web-based Conferencing: Tips, Tricks, and Scenario-based Best Practices for Clinicians During a Pandemic Crisis. Journal of Computer Assisted Tomography. 2020;44(4):465-71.

12. Li CH, Rajamohan AG, Acharya PT, Liu C-SJ, Patel V, Go JL, et al. Virtual read-out: radiology education for the 21st century during the COVID-19 pandemic. Academic Radiology. 2020.

13. Slanetz PJ, Parikh U, Chapman T, Motuzas CL. Coronavirus Disease 2019 (COVID-19) and Radiology Education-Strategies for Survival. Journal of the American College of Radiology. 2020;17(6):743.

14. Henderson D, Woodcock H, Mehta J, Khan N, Shivji V, Richardson C, et al. Keep calm and carry on learning: using Microsoft teams to deliver a medical education programme during the COVID-19 pandemic. Future healthcare journal. 2020;7(3):e67.

15. Martin L, Tapp D. Teaching with Teams: An introduction to teaching an undergraduate law module using Microsoft Teams. Innovative Practice in Higher Education. 2019;3(3).

16. Shalini Shah M, Sudhir Diwan M, Lynn Kohan M, David Rosenblum M, Christopher Gharibo M, Amol Soin M, et al. The technological impact of COVID-19 on the future of education and health care delivery. Pain physician. 2020;23:S367-S80.

17. Bhargava P, Dhand S, Lackey AE, Pandey T, Moshiri M, Jambhekar K. Radiology education 2.0-on the cusp of change: part 2. eBooks; file sharing and synchronization tools; websites/teaching files; reference management tools and note taking applications. Academic radiology. 2013;20(3):373-81.

18. Rayhan RU, Zheng Y, Uddin E, Timbol C, Adewuyi O, Baraniuk $\mathrm{JN}$. Administer and collect medical questionnaires with Google documents: a simple, safe, and free system. Appl Med Inform. 2013;33(3):12-21.

19. Assadi R, Gasparyan AY. Editing, Publishing and Aggregating Video Articles: Do We Need a Scholarly Approach? J Korean Med Sci. 2015;30(9):1211-2.

20. Wijesooriya NR, Mishra V, Brand PL, Rubin BK. COVID-19 and telehealth, education, and research adaptations. Paediatric Respiratory Reviews. 2020.

21. Kali A. Reference management: A critical element of scientific writing. J Adv Pharm Technol Res. 2016;7(1):27-9.

22. ySungur MO, Seyhan TO. Writing references and using citation management software. Turk J Urol. 2013;39(Suppl 1):25-32.

23. Prior F, Almeida J, Kathiravelu P, Kurc T, Smith K, Fitzgerald TJ, et al. Open access image repositories: high-quality data to enable machine learning research. Clin Radiol. 2020;75(1):7-12.

24. Dhawan S. Online learning: A panacea in the time of COVID19 crisis. Journal of Educational Technology Systems. 2020;49(1):5-22.

25. Alpert JB, Young MG, Lala SV, McGuinness G. Medical Student Engagement and Educational Value of a Remote Clinical Radiology Learning Environment: Creation of Virtual Read-Out Sessions in Response to the COVID-19 Pandemic. Academic Radiology. 2020.

26. Vardeny O, Hernandez AF, Cohen LW, Franklin A, Baqai M, Palmer S, et al. Transitioning to the National Institutes of Health single institutional review board model: Piloting the use of the Streamlined, Multi-site, Accelerated Resources for Trials IRB Reliance. Clin Trials. 2019;16(3):290-6.

27. Christian MC, Goldberg JL, Killen J, Abrams JS, McCabe MS, Mauer JK, et al. A central institutional review board for multiinstitutional trials. N Engl J Med. 2002;346(18):1405-8.

28. Klinger C, Landeg O, Murray V. Power outages, extreme events and health: a systematic review of the literature from 2011-2012. PLoS Curr. 2014;6.

Publisher's Note Springer Nature remains neutral with regard to jurisdictional claims in published maps and institutional affiliations. 